\title{
Tabularia
}

TABULARIA Sources écrites des mondes normands médiévaux

\section{Carmen de fundatione, ruina et restauratione inclyti monasterii gemmeticensis}

Pierre Bouet

\section{OpenEdition}

\section{Journals}

Édition électronique

URL : http://journals.openedition.org/tabularia/2843

DOI : 10.4000/tabularia.2843

ISSN : 1630-7364

Éditeur :

CRAHAM - Centre Michel de Boüard, Presses universitaires de Caen

\section{Référence électronique}

Pierre Bouet, "Carmen de fundatione, ruina et restauratione inclyti monasterii gemmeticensis »,

Tabularia [En ligne], Sources en ligne, mis en ligne le 29 décembre 2001, consulté le 01 mai 2019. URL http://journals.openedition.org/tabularia/2843; DOI : 10.4000/tabularia.2843

Ce document a été généré automatiquement le 1 mai 2019.

CRAHAM - Centre Michel de Boüard 


\title{
Carmen de fundatione, ruina et restauratione inclyti monasterii gemmeticensis
}

\author{
Pierre Bouet
}

1 Ce poème de 185 vers en hexamètres dactyliques fut composé vraisemblablement au XVI siècle par un moine de Jumièges, dont un manuscrit nous dit qu'il s'appelait Adrien¹.

\section{Résumé du poème}

2 Le Carmen présente une brève histoire du monastère de Jumièges. Dans l'«argument " l'auteur invite le voyageur avisé (prudens uiator, 1) à s'arrêter pour contempler les tableaux où sont représentés les heurs et malheurs du monastère de Jumièges. Après avoir proposé un étymologie du mot Gemmeticus, il annonce les trois tableaux ou groupes de tableaux : fondation du monastère, destruction par les Daces et restauration. Le poète compose ce carmen pour évoquer brièvement les faits représentés sur ces tableaux.

3 La fondation par Philibert, venu de la « Bourgogne celtique " (Celtica... Burgundia, 18), est datée de 640 (saecula sex... cum lustris... octo, 16). Philibert s'installa avec ses moines dans un site sauvage où les forêts pleines de ronces le disputaient aux marécages (spinosis horrida syluis, undique... cincta palude, 21-22). Or cet endroit qui n'avait connu ni Cérès ni Bacchus devint l'une des régions les plus fertiles au monde (toto foecundior extititorbe, 25). Là où vivaient des bêtes sauvages solitaires, s'élevèrent bientôt des églises étincelantes d'or où jour et nuit les moines, à l'imitation des chœurs angéliques, faisaient retentir des chants sacrés en l'honneur du Tout-Puissant.

4 À la place des ifs malodorants, de l'aconit et de la ciguë, la terre se mit à produire « la baie noire, les raisins pourpres et les douces moissons " (bacca nigra... uuae purpurae... dulces aristas, 41-43). Les prairies aux fleurs multicolores et les gras pâturages remplacèrent les marais couverts de roseaux. A la marée montante la Seine regorgeait de poissons venus 
de la mer et, à cause du reflux, les rivières fertilisaient le sol par les inondations, comme le Nil en Egypte. C'est le Créateur du monde (Sator rerum, 54) qui veille à l'équilibre des océans et de la terre et à la survie des animaux terrestres, des oiseaux du ciel et des espèces marines. Par ses dons, qui rappellent ceux qu'il fit au peuple hébreu dans le désert, le Tout-Puissant a transformé une âpre solitude en une terre très fertile (aspera quae fuerat, fieret cultissima eremus, 73).

5 L'arrivée des Daces avec le barbare Hasting vers 840 conduit à l'anéantissement de la France du nord et notamment à la destruction des églises où était célébré le culte du Très Haut, du Dieu Tonnant (altithroni... tonantis, 83). Remontant avec leurs navires le cours de la Seine, les Daces ont assailli la Neustrie et provoqué la désolation (tunc misero miscetur Neustria luctu, 88): les moines de Jumièges ont fui à Haspres, une de leurs dépendances (Haspreadum deserta petunt ubi claustra domusque/nostri iuris erant, 92-93), pour ne pas manquer à leurs vœux. Certains cependant ont décidé de rester avec la volonté de résister par la force aux ennemis. Mais, au terme d'un siège impitoyable, les Daces ont réussi à pénétrer dans l'enceinte monastique qu'ils ont pillée, rasée et incendiée. L'abbatiale dédicacée à Notre-Dame a tenu plus longtemps, mais, sous les coups répétés des barbares, elle a fini par "gémir et par s'écrouler dans un nuage de poussière " : «la terre percutée par cette masse énorme a tremblé » (et concussa tremit tam uasto pondere tellus, 124). Pillage et massacre ne prirent fin que lorsque tout fut la proie des flammes.

6 Un siècle après, "Cérès finit par apaiser les colères de Mars » (Ceres Martis compescuit iras, 131). Deux des pères exilés revinrent vers 940 à Jumièges, qu'ils découvrirent couvert de broussailles et de buissons où se terraient les bêtes sauvages. Dans leur prière les deux ermites sollicitèrent la pitié du Très Haut et son secours pour relever l'église de ses ruines et rétablir le culte. C'est le duc Guillaume, issu du sang de Rollon, qui fut l'artisan de cette restauration. La venue fortuite du duc lors d'une partie de chasse le met en présence des deux ermites qui lui offrirent le pain et l'eau dont ils se nourrissaient habituellement. En refusant de partager ce maigre repas (dux uero aduersus tam uilia fercula cedit/protinus hisce locis, 165-166), le duc s'attira la colère de Dieu : la charge furieuse d'un sanglier le ramène à plus de sagesse. Prenant conscience de l'offense faite aux deux ermites (at memor offensae, 172), Guillaume revient sur les lieux et promet de reconstruire le monastère. Les artisans du duc restaurent le monastère sur un plan plus réduit (minori schemate, 181-182) et, peu après, l'abbatiale relevée de ses ruines est à nouveau consacrée à la Vierge.

\section{Les manuscrits}

Nous connaissons le poème par trois manuscrits de la Bibliothèque nationale de France, deux du XVII ${ }^{e}$ siècle et un du début du XVIII ${ }^{e}$ siècle :

1. le manuscrit latin 12778 (XVII ${ }^{\mathrm{e}}$ siècle) comprend 398 pages. C'est l'ancien Saint-Germaindes-Prés $n^{\circ} 578$ (Miscellanea monastica, D-L); le poème se trouve aux pages 153-155. Selon M. Nortier, les documents qui composent ce manuscrit ont été réuinis par Dom Le Michel, Chantelou et Mabillon ${ }^{2}$.

2. le manuscrit des collections diverses Baluze $n^{\circ} 58$ (XVII siècle) consacre 262 pages à des extraits de chroniques ou de textes relatifs à la Normandie. Selon M. Nortier, ce manuscrit rassemblerait les papiers d'André Duchesne qui donna, au début du XVII siècle, les premières éditions des chroniqueurs normands des $\mathrm{XI}^{\mathrm{e}}$ et $\mathrm{XII}{ }^{\mathrm{e}}$ siècles. C'est dans l'incipit de ce poème, qui occupe les pages $174-176 \mathrm{v}$, que A. Duchesne considère le moine Adrien comme l'auteur de ces 185 vers $^{3}$. 
3. le manuscrit fr 4899 de 512 pages constitue un recueil de documents concernant les abbayes normandes. Les textes relatifs à l'abbaye de Jumièges sont présentés de la page 255 à la page 293: ils forment un ensemble intitulé «Mémoires des antiquités et choses plus mémorables de l'abbaye Saint-Pierre de Jumièges, ordre de saint Benoît, congrégation de Saint-Maur ». Il fut composé par le "père antiquaire » à la demande du prieur Dieudonné Buisson et adressé à $\mathrm{M}$. de Foucault au début du XVIII ${ }^{\mathrm{e}}$ siècle. Le poème commence le recueil (p. 261-266).

\section{Poème et fresques du cloître}

Ce manuscrit fr 4899 (p. 261) nous apprend que le poème est une copie « des vers latins gravés sur des plaques de cuivre posées à l'entrée du cloitre ». Ce même manuscrit nous informe (p. 266) qu'un autre texte en vers était gravé sur une plaque de cuivre placée à côté du maître-autel. Ce texte évoquait les deux dédicaces de l'église abbatiale, celle de 1067 et celle de 1278 (pour le nouveau chœur gothique). Le titre du poème dans ce manuscrit du XVIII ${ }^{e}$ siècle se termine par cette indication : carmen cuius claustri parietibus inhaerent tabellae quae totius rei summam indicant (« poème dont les panneaux qui racontent la totalité de l'histoire (du monastère) sont fixés aux murs du cloitre »).

La reprise du terme tabella au début de l'argument du poème fait difficulté : le poète invite le " passant avisé » à arrêter sa marche résolue et à contempler « ce qui est peint/ décrit sur ces tableaux parfaitement dessinés/gravés » (prudens uiator/aspice quae graphicis sunt his depicta tabellis, v. 1-2). Que signifie l'expression graphicis tabellis? S'agit-il des panneaux de cuivre où sont gravés les vers latins et qui sont fixés aux murs du cloitre? Ou des tableaux peints que le poète invite à regarder et dont le poème présente un bref commentaire? Il est difficile de trancher dans la mesure où les termes latins graphicis picta tabellis peuvent se comprendre au sens propre (peinture) ou au sens figuré (texte gravé).

Pourtant l'existence de fresques sur les murs du cloître lève, nous semble-t-il, toute ambiguïté : le poème gravé sur les plaques de cuivre apparaît bien comme le commentaire littéraire des peintures qui se trouvaient dans le cloître. D'ailleurs le contexte lexical des termes qui continuent l'invitation du poète fait référence au domaine visuel: aspice « regarde » et non lege « lis", spectantis in unda seu leui in speculo solet apparere figura, «le visage de celui qui regarde apparaît habituellement sur la surface de l'eau ou sur le miroir poli ». L'emploi du terme depicta confirme cette interprétation.

11 À propos de ces peintures du cloître de Jumièges, nous disposons d'un témoignage intéressant. Un notaire de Jumièges, du nom de Deshayes, publia en 1821, un petit opuscule intitulé $\mathrm{La}$ Terre gémétique où est longuement décrit le cloître avant sa destruction survenue vers les années 1797-1802. Le notaire déclare notamment : « Quatre fresques, à la vérité très grossières, mais qui sous ce rapport n'en étaient que plus précieuses en ce qu'elles portaient avec elles l'empreinte du siècle reculé qui les vit naître et révélaient l'enfance où l'art était alors, y représentaient la première, la punition des Énervés; la seconde, la révélation faite à saint Aychadre que Dieu devait rappeler à Lui une partie de ses religieux; la troisième, le débarquement des Danois et l'incendie de Jumièges en 840 ; et la quatrième, la rencontre que fit Guillaume Longue Épée de deux religieux qui lui offrirent les aliments grossiers dont ils faisaient usage. $»^{4}$ Dans un ouvrage ultérieur, ce même auteur décrit plus longuement la troisième fresque: «L'abbaye s'y trouvait représentée, mais infidèlement, avec les deux clochers du portail 
et la grand pyramide ou aiguille qui s'élevait sur l'entrée du chœur. On y voyait en outre les religieux fuir en enlevant leurs richesses; l'abbé marchait à leur tête et portait un ostensoir. Le feu était aux quatre coins de l'abbaye, dont les flots venaient battre les murs. On y voyait une grande partie des Danois se jetant à l'eau pour débarquer. D'autres, à peu de distance, escaladaient les murs ou lançaient des brandons enflammés sur les édifices. » L'auteur ajoute que ces fresques « ont dû être faites postérieurement au gouvernement de l'abbé François de Fontenai $» .^{5}$

Ce témoignage concerne le cloître qui fut édifié par l'abbé François de Fontenay et achevé peu avant 1530. C'était un édifice particulièrement décoré où se mêlaient le style gothique flamboyant et le style Renaissance. Cette reconstruction était conforme à la volonté réformatrice de l'abbé Philippe de Luxembourg, un des prédécesseurs de François de Fontenay, qui avait rétabli en 1515 la discipline monastique en plaçant l'abbaye de Jumièges sous l'autorité de l'abbaye berrichonne Chezal-Benoît. Ce nouveau cloître de 1530 était disposé sur le flanc sud de la nef de l'abbatiale Notre-Dame : il remplaçait le cloître qui avait été construit par l'abbé Guillaume en 1349 et qui, lui-même, s'était substitué au premier cloître édifié au XI ${ }^{e}$ siècle ${ }^{6}$.

\section{Date de composition}

Les fresques du XVI siècle, que vit le notaire Deshayes avant leur destruction, furentelles à l'origine de la composition du poème? On peut en émettre l'hypothèse pour plusieurs raisons. Le texte du poème correspond bien aux deux derniers tableaux décrits par Deshayes (l'incendie de Jumièges par les Danois et la rencontre du duc Guillaume Longue Épée avec les deux ermites de Jumièges). Cependant le début du poème consacré à la fondation du monastère par saint Philibert ne correspond à aucune des deux premières fresques (la punition des Énervés et la vision de saint Aycadre). Peut-être y avait-il sur les murs du cloître au XVI ${ }^{e}$ siècle d'autres fresques que les accidents de l'histoire ont fait disparaitre. On conçoit mal, en effet, un programme iconographique sur les grands moments du monastère sans l'évocation du saint fondateur transformant ce site sauvage en une terre d'élection.

On pourrait imaginer que le programme iconographique du XVIe siècle se soit inspiré de ce poème composé en des temps plus anciens : c'est la position qu'avaient adoptée la plupart des critiques qui ont daté la composition du Carmen du milieu du $\mathrm{X}^{\mathrm{e}}$ siècle et qui en ont fait une production contemporaine du Planctus sur la mort de Guillaume Longue Épée en 943. Plus récemment Elisabeth Van Houts a proposé une date plus récente, peu après 1024, pour la composition du Carmen de Jumièges : elle y voit un texte rédigé dans une langue artificielle à la manière de Dudon de Saint-Quentin ${ }^{7}$. Or, une comparaison des expressions les plus originales de notre Carmen avec le De moribus et actis primorum Normanniae Ducum de Dudon ${ }^{8}$ montre de façon très claire que la langue de ces deux auteurs n'ont pratiquement aucun point commun : c'est le même souci de préciosité, mais ce sont deux sources d'inspiration différente. Nous avons cependant trouvé quelques rares analogies :

\begin{tabular}{|l|l|l|l|}
\hline & Carmen & & Dudon de Saint-Quentin \\
\hline $12:$ & ...Daciae Phalanges & $3,64:$ & ...gentis utriusque phalanx \\
\hline $137:$ & ...Panomphaeo Tonanti & $1,7:$ & ...astra Tonantis \\
\hline
\end{tabular}




\begin{tabular}{|l|l|l|l|}
\hline $101:$ & ...undas sulcando & $4,124:$ & ...sulcaui profunda aequora \\
\hline $89-90:$ & ...horror ingruit armorum & $2,9:$ & ...horrore armorum ingruente (Virgile) \\
\hline $101:$ & ...fluctiuagas undas & $1,5:$ & ...fluctiuagum pelagus \\
\hline
\end{tabular}

En revanche aucun écho chez Dudon de tout un ensemble d'expressions caractéristiques du Carmen : monoptolemi, Lelegum turmae, Phoebus uoluerat, Virginis a partu, fundamina templi, dei capripedes, soliuagis feris, stellantis Olympi, taxus olens, iunci lacustres, gemmantia prata, turgente salo, mirante polo, incunabula Christi, sitibundus, uexilla Gradiui, mystae, Stygiae morti, sacra spurcanda, expansis alis, glomeratis turmis, rigidis contis, patulas fenestras, semirutis tectis, Christipara uirgo, camerato culmine, in uepres redigi, latebrosa lustra, penetralia syluae densae, numinis impulsu, encoenia templi, etc.

Nous disposons de plusieurs indices qui nous incitent à placer la composition du poème $\mathrm{au} \mathrm{XVI}^{\mathrm{e}}$ siècle. Les allusions mythologiques révèlent un esprit averti des subtilités généalogiques des dieux et des déesses à la manière des intellectuels de la Renaissance. En outre le poète a une préférence pour les mots rares et en particulier pour les termes empruntés au grec. Le mot mysta, « initié aux mystères » se rencontre chez Ovide, Sidoine Apollinaire et Rufin ${ }^{9}$, mais, chez ces auteurs, il est très rarement employé comme synonyme de monachus. Il en est de même pour sitibundus, « assoiffé » qu'on ne rencontre que chez Venance Fortunat et Paul Diacre ${ }^{10}$ et Christipara, «Mère du Christ » qui n'est attesté que dans un poème liturgique ${ }^{11}$. Le mot panomphaeus n'a été employé durant toute la latinité antique et médiévale que par Ovide dans une tournure dont s'est inspiré notre auteur : Ovide évoque un antique autel consacré à Jupiter « Tonnant de qui émanent tous les oracles ${ }^{12}$ (ara panomphaeo uetus est sacrata Tonanti, cf. Carmen:... panomphaeo fuerant sacrata Tonanti, 137). Il crée même un hapax monoptolemos à partir de deux termes grecs monos et ptolemos qui a vraisemblablement le sens d'« athlète " par référence au gymnase d'Athènes Ptolemaios.

17 La manière de dater les événements constitue un indice encore plus sûr dans la mesure où le procédé utilisé par l'auteur du Carmen est étranger aux habitudes antiques et médiévales. Par deux fois, en effet, le poète normand a recours à une datation exprimée en siècles et en lustres par référence à la naissance du Christ :

16 - 17 : Saecula sex Phoebus cum lustris uoluerat octo/Virginis a partu...

«Phébus avait déroulé six siècles ( 600 années) et huit lustres ( 8 x 5 années) depuis

l'enfantement de la Vierge... »

76 - 77 : Lustra octo totidem labentia saecula cursu/...post incunabula Christi...

"les astres avaient parcouru huit lustres ( 8 × 5 années) et autant de siècles

(800 années) après la naissance (le berceau) du Christ... »

Cette manière de calculer les durées en siècles de 100 ans ne serait apparu qu'au XVI siècle si l'on en croit J. Le Goff ${ }^{13}$. Il est vrai que le mot saeculum en latin classique et médiéval a plusieurs significations: "la génération", puis "durée d'une génération humaine » (environ 33 ans selon Cicéron); au singulier saeculum peut correspondre à une "durée de 100 ans », mais au pluriel il exprime "une longue durée », "la durée de ce monde » ou «l'éternité ». Les Chrétiens useront de ce terme pour signifier «le monde terrestre », "les hommes» ou " la vie païenne de ce monde». Jamais le terme saecula n'est utilisé pour exprimer un multiple de 100 années par référence à un fait précis (fondation de Rome ou naissance du Christ). 
19 Ainsi, il nous semble vraisemblable que la réalisation des fresques sur les murs du cloître dans la première moitié du XVI ${ }^{e}$ siècle a conduit un moine nommé Adrien a composé un poème de 185 vers qui, par la suite, a été gravé sur des plaques de cuivre posées à l'entrée de ce cloître afin d'aider les visiteurs à saisir le sens de ces scènes iconographiques. D'ailleurs le cas de Jumièges n'est pas isolé : il était alors à la mode de mettre en image l'histoire des origines. La réalisation des fresques de Jumièges est sans doute à rapprocher des vingt-huit tapisseries sur l'histoire du Précieux-Sang que l'abbé Antoine Boyer (1505-1519) offrit à son abbaye de Fécamp ${ }^{14}$.

\section{Étude linguistique}

20 La lecture, même rapide, de ce poème révèle qu'il s'agit d'un exercice littéraire un peu gratuit. L'auteur s'attarde peu sur les particularités historiques du monastère de Jumièges qu'il emprunte à la tradition et notamment à Guillaume de Jumièges. Ce qui l'intéresse c'est de composer une poésie champêtre à la manière de Virgile et d'Ovide, en développant le topos que l'on rencontre dans la plupart des histoires de fondations monastiques: la solitude où s'installent les moines fondateurs est une terre sauvage et stérile qui par la bienveillance divine (et le travail acharné des moines) se transforme en un véritable éden.

21 Une étude rigoureuse mettrait en évidence tous les emprunts textuels faits aux poètes antiques, profanes ou chrétiens. Au premier plan se trouve Virgile dont l'influence est manifeste dans tout le Carmen, que cette influence soit directe ou qu'elle s'effectue par le biais d'autres poètes imitateurs de Virgile ou par la médiation des ouvrages de grammaire. Il est instructif de mettre en évidence ces correspondances du Carmen avec le poète de Mantoue.

\begin{tabular}{|l|l|l|l|}
\hline \multicolumn{2}{|l|}{ Carmen } & \multicolumn{2}{l|}{ Virgile } \\
\hline 1 & siste gradum & E 6, 645 & siste gradum \\
\hline 13 & a sedibus imis & E 1,84 & a sedibus imis \\
\hline 19 & stipante caterua & E 1, 497 & stipante caterua \\
\hline 21 & quondam spinosis horrida siluis & E 8, 348 & olim siluestris horrida dumis \\
\hline 41 & Alcinoi siluae & G 2,87 & Alcinoi siluae \\
\hline 46 & uario colore prata & E 5, 89 & uario colore \\
\hline 62 & secent liquidum aera pennis & Ci 541 & secat aera pinnis \\
\hline 64 & aetherea uescitur aura & E 1,546 & uescitur aura aetheria \\
\hline 76 & lustra labentia & E 1,281 & lustris labentibus \\
\hline 81 & gelidam Arcton & E 6,16 & gelidas ad Arctos \\
\hline 86 & intrat portus & E 3, 254 & intrare portus \\
\hline 86 & fama per urbes uolat & E 8, 554 & fama uolat per urbem \\
\hline $86-87$ & fama, tantae praenuntia cladis & E 11,139 & fama, tanti praenuntia luctus \\
\hline $89-90$ & horrror ingruit armorum & E 2, 301 & horor ingruit armorum \\
\hline
\end{tabular}




\begin{tabular}{|l|l|l|l|}
\hline 102 & ipsis allabitur oris & E 6,2 & allabitur oris \\
\hline 107 & pandere portas & E 12,584 & pandere portas \\
\hline 108 & rigidis extrudere contis & E 9,510 & duris detrudere contis \\
\hline 110 & acta testudine & E 2, 441 & acta testudine \\
\hline 116 & nullo discrimine & E 1,574 & nullo discrimine \\
\hline 117 & miscent incendia & E 2,329 & incendia miscet \\
\hline 124 & $\begin{array}{l}\text { concussa tremit tam uasto } \\
\text { pondere tellus }\end{array}$ & E 9,752 & $\begin{array}{l}\text { ingenti concussa est pondere } \\
\text { tellus }\end{array}$ \\
\hline 126 & accincti praedae & E 1,210 & praedae accingunt \\
\hline 129 & medios in ignes & E 11,787 & medium per ignem \\
\hline 136 & lustra ferarum & E 3,647 & lustra ferarum \\
\hline 153 & summo Olympo & E 11,726 & summo olympo \\
\hline 153 & nec mora & E 12, 553 & nec mora \\
\hline 154 & sanguine cretum & E 4, 191 & sanguine cretus \\
\hline
\end{tabular}

22 Parfois l'emprunt court sur plusieurs vers comme le passage qui concerne la destruction de l'abbatiale Notre-Dame mis en rapport avec la destruction de la ville de Troie dans une comparaison avec l'abattage d'un orne antique:

\begin{tabular}{|l|l|}
\hline Carmen 120-124 & Virgile, Enéide, 2, 226-231 et 9,752 \\
\hline $\begin{array}{l}\text { Quam ferro accisam quatiunt et uerbere } \\
\text { crebro }\end{array}$ & $\begin{array}{l}\text { Cum ferro accisam crebrisque bipennibus } \\
\text { instant }\end{array}$ \\
\hline $\begin{array}{l}\text { Fundamenta cauant : ast uulnere uicta } \\
\text { supremo }\end{array}$ & Eruere agricolae certarim... \\
\hline $\begin{array}{l}\text { Ingemuit traxitque iugo conuulsa ruinam. } \\
\text { Tollitur in caelum densato puluere nubes : }\end{array}$ & Congemuit traxitque iugis auolsa ruinam... \\
\hline Et concussa tremit tam uasto pondere tellus. & ...Tellus concussa est ingenti pondere. \\
\hline
\end{tabular}

Certaines pages de Virgile ont plus particulièrement inspiré le poète du Carmen. Le livre II des Géorgiques (vers 85-160) donne tout un ensemble de termes relatifs à la campagne que l'on retrouve dans l'évocation du site de Jumièges après le travail des moines (vers 37-52) : bacca (G 2, 85), Alcinoi siluae (86), uites (91), pinguibus terris (93), purpurae (95), tumidis racemis (102), taxi (113), olentia (134), grauidae fruges (143), aconita (157). Mais le poète a des réminiscences instinctives, fruit de sa formation scolaire et de ses lectures. Certaines expressions sont plutôt des créations à la manière de Virgile ou d'Ovide que des emprunts véritables.

\begin{tabular}{|l|l|l|}
\hline & Carmen & Virgile \\
\hline
\end{tabular}




\begin{tabular}{|l|l|l|}
\hline 42 & purpurae uuae & purpurae uite \\
50 & turgente salo & superante salo \\
57 & tumidis undis & tumida aequora \\
$78-81$ & impetiit litora & littora petunt \\
84 & infesto Marte & Marte aduerso \\
85 & classibus instructis & nauibus instructis \\
139 & ad sidera uertunt & euertunt ad sidera \\
\hline
\end{tabular}

Le caractère artificiel et scolaire du Carmen se manifeste également dans l'abondance des allusions mythologiques. Le poète ne se contente pas de l'évocation traditionnelle des dieux et des déesses pour désigner des réalités prosaïques comme Cérès $(24,132)$ pour les moissons, Thétis (57) pour la mer, Mars $(84,90,132)$ pour la guerre et Phébus $(16)$ pour le soleil. Certaines allusions, plus subtiles, révèlent une connaissance très précise de la mythologie et des généalogies divines, à l'école d'Ovide. Dieu, créateur du monde, est constamment comparé à Jupiter : Il contemple le monde du sommet de l'olympe (summo spectat Olympo, 153), Il est le Tonnant (summo tonanti, 33, 138), que l'on invoque en tous lieux (panomphaeo, 138) et qui siège sur un trône élevé (altithroni, 83). Diane est présentée comme la fille de Latone/Léto et la descendante des Titans : Latoïs (48), Titania (49). Quant à Bacchus on le qualifie de Lyaeus, forme latinisée qui vient du grec et qui signifie « celui qui libère des soucis ", tour habituel chez Ovide et Horace. Le poète du Carmen emprunte également des termes rares aux cycles légendaires antiques : Eous (7) pour l'Orient, Arcton (81) pour le nord, Alcinoi (41) pour les vergers, Laniger (131) pour la constellation du Bélier.

La relation du Carmen à l'histoire est, dans cette perspective, réduite à sa plus simple expression. Le poète n'ignore pas les Gesta Normannorum Ducum de Guillaume de Jumièges comme le montre un certain nombre d'expressions. Il reprend l'étymologie du mot Gemmeticus qui viendrait, selon Guillaume de Jumièges, du terme gemma (quidam a gemma illum [Gemmeticum] fore uocatum arbitrantur), sans toutefois en retenir le commentaire: "Jumièges brille par son site et l'abondance de ses productions comme une pierre sur un anneau $»^{15}$. Si l'auteur du Carmen fait allusion aux 900 moines qui vivaient à Jumièges en s'inspirant des Gesta (in nogentorum monachorum numero), il ne parle pas du rôle joué par la reine Bathilde (opitulante regina Bathilde) ${ }^{16}$, préférant évoquer le nom du roi Dagobert ( Dagoberti munere regis, 26).

Mais dans les vers qui racontent la restauration du monastère à partir des années 940 , le poète reprend plusieurs expressions du chroniqueur $\mathrm{du}_{\mathrm{XI}}{ }^{\mathrm{e}}$ siècle, textuellement ou par synonymie comme le montre le tableau suivant.

\begin{tabular}{|l|l}
\hline Carmen, 153-181 & Guillaume de Jumièges, III, 7
\end{tabular} 


\begin{tabular}{|c|c|}
\hline $\begin{array}{l}\text { Guillelmumque ducem, } \\
\text { Rollonis sanguine cretum } \\
\text { Coelitus huc afflat } \\
\text { uenando flectere cursum, } \\
\text { Qui monachos nactus } \\
\text { scitari coepit ab illis: } \\
\text { Quis tam decrepitos istis } \\
\text { uos appulit oris ?... } \\
\text { Quae structurae inerant... } \\
\text { Cumque rei seriem... } \\
\text { ordine narrassent... } \\
\text { Dux uero aduersus tam } \\
\text { uilia fercula... } \\
\text { Seminanimemque ducum } \\
\text { culmis prostruit acutis. } \\
\text { At memor offensae mox } \\
\text { uiribus ipse resumptis } \\
\text { Hos repetiit Patres... } \\
\text { Sumpturusque cibum } \\
\text { quem spreuerat accubat } \\
\text { Structuras renouare } \\
\text { parant arctando minori... }\end{array}$ & $\begin{array}{l}\text {... Quos cum idem dux Willelmus uenatus gratia super-ueniens illic } \\
\text { reperisset, percunctari ab eis cepit, quibus ab oris illuc aduenissent } \\
\text { uel quae tam praecipua edificia forent. } \\
\text { Cui interroganti rei seriem illico serui Dei per ordinem detexerunt } \\
\text { caritatemque panis ordeaci et aquae obtule runt. Quam cum ob } \\
\text { vilitatem panis et aquae sumere contempsisset... } \\
\text {... Porro aper... eum prostratum uehementer attriuit. Qui post } \\
\text { paululum sensu recepto a sana mente mona chos repetiit et caritatem } \\
\text { quam incaute spreuerat, ab eis recipiens locum se restauraturum } \\
\text { spopondit. } \\
\text {... Claustrum uero et cuncta receptacula muniens a sui magnitudine } \\
\text { breuiata habitacula reddidit. }\end{array}$ \\
\hline
\end{tabular}

Ainsi sous ces artifices littéraires et ces allusions savantes peuvent apparaître des informations qui montrent chez le moine Adrien un souci historique : peut-être avait-il accès à des sources aujourd'hui disparues. Si le premier tableau consacré à saint Philibert apparait un jeu bucolique, les vers qui racontent le débarquement des Danois et la destruction du monastère fournissent des informations de quelque intérêt: fuite des moines à Haspres où la communauté possède domos et claustra, attaque des portes, escalade des murs, sape des remparts, recherche du butin et destruction totale des édifices.

\section{Carmen de fundatione, ruina et restauratione inclyti monasterii gemmeticensis}

\section{Argumentum}

Siste gradum stabilem, prudens quicunque uiator: Aspice quae graphicis sunt his depicta tabellis; En tibi clara patent, sicut spectantis in unda, Seu leui in speculo solet apparere figura, Quae fuerint huius rutilae primordia gemmae (Gemmeticum siquidem a gemma dixere priores, Quod reliquis gemmae praecelleret instar Eoae). Inde scies quinam dederint regesque ducesque, Tot bona, tot terras et opes, tot praedia, census, 10 Vnde monoptolemi nongenti, caelitus acti, 
Strictius inclusam possent hic ducere uitam.

Quas itidem, Lelegum turmae Dacaeque phalanges,

Intulerint clades, qui tandem a sedibus imis

Haec loca sarcierint priscis conuulsa ruinis,

Expedio breuibus complexens gesta camoenis.

\section{Prima fundatio dicti monasterii}

Saecula sex Phoebus cum lustris uoluerat octo Virginis a partu, dum sanctus uir Philibertus, Celtica quem genuit claro Burgundia partu, Hos petiit fines, tanta stipante caterua 20 Quanta Jacob Gessen ipsam culturus eremum.

Quae licet haec quondam spinosis horrida syluis, Vndique diuersa restagnans cincta palude, Gente prius nulla, nullis sit culta colonis, Nec Cereri penitus, nec dulci nota Lyaeo, Nulla tamen toto foecundior extitit orbe, Ille pater postquam Dagoberti munere regis Prima huius sacri statuit fundamina templi: Mox quippe antiquam mutarunt omnia formam. Hic ubi capripedum latitabant antra deorum 30 Soliuagis habitata feris, nunc peruia sanctis, Aurea templa nitent, instar stellantis Olympi. Nocte dieque preces effundunt, inclyta diuum Cantica nunc resonant, nunc summo sacra Tonanti, Agmina persoluunt monachorum munia laudum, Angelicos imitata choros, ubi monstra ferarum Horrisonis uacuas complebant uocibus auras. Quae prius exsuccos syluestri germine foetus Tristis alebat humus, condensis obsita dumis, Fertilis ambrosios coepit producere fructus. 40 Taxus olens, tiliae molles, aconita, cicutae Alcinoi syluis cedunt; nigra bacca racemis, Purpureae grauidis turgent in uitibus uuae, Et renouatus ager dulces producit aristas. Ilic ubi crescebant calami iuncique lacustres, Luxuriant uario gemmantia prata colore: Deque paludigenis nunc surgunt pinguia cannis Pascua, et ingentes labuntur ab aequore pisces Fluctibus impulsi, quoties Latois utrinque Iungit horizonti roseos Titania currus; 50 Ac rapidus, turgente salo, reflectitur amnis, Ut refluant per agros, per prata uirentia riui, Qui foecunda suis irrorent frugibus arua, $V t$ rigat Aegyptum septeno gurgite Nilus. Haec dedit ille Sator rerum, qui terminat orbem, Maxima qui solidam statuit super aequora terram, Sistit et immensos praescriptis finibus amnes, Ne Cybele tumidis obruatur Thetios undis. Imbribus et montes sterilesque humectat arenas, $V t$ sua cunctiparens concedat munera tellus, 60 Vnde trahant uitam quaecunque cibaria poscunt: Siue per incultos errent animalia campos, Siue secent liquidum stridentibus aera pennis, Vel quae monstra natant uariis distincta figuris. 
Quidquid et aetherea spirando uescitur aura,

Confouet, et propria unicuique alimenta ministrat.

Denique qui quondam siccis de rupibus undas

Duxit, et ex alto demisit uertice carnes

Mannaque, seu pluuio funduntur ab aethere nimbi

$V t$ populum, mirante polo, nutriret Hebraeum,

70 Per steriles campos desertaque rura uagantem.

Sic sua Gemmeticis largitus dona colonis

Fecit, ut inuitis naturae legibus olim

Aspera quae fuerat, fieret cultissima eremus:

Quam circumflexo praecingit Sequana fluxu,

Haud secus ac Arcton sinuosi cauda draconis.

\section{Eiusdem monasterii destructio}

Lustra octo totidem labentia saecula cursu Astra penetrarant post incunabula Christi, Belgica dum Getico Francorum littora bello, Barbarus Hastingus, Dacis egressus ab oris, 80 Haud secus atque leo praedae sitibundus ab antro, Impetiit, gelidam qua Francia respicit Arcton, Finitimasque urbes et plurima castra subegit. Proh scelus! Altithroni celeberrima templa Tonantis Diruit, infesto peruertens omnia Marte.

Classibus instructis tandem, Boreaque secundo, Sequanicos intrat portus: cito fama per urbes Peruolat attonitas, tantae praenuntia cladis. Vndique tunc misero miscetur Neustria luctu, Vndique bella premunt, hostes grassantur, et horror 90 Ingruit armorum, fulgent vexilla Gradivi. Hinc procul aufugiunt monachi : procul abdita mystae Haspreadum deserta petunt, ubi claustra domusque, Nostri iuris erant, ne sacrum soluere uotum, Aut efflare animam urgerentur, et obruta diris Corpora tormentis Stygiae dimittere morti. Pars tamen hic sistunt armis, et milite complent Haec loca, quo possent hostes arcere minaces: Duxerunt satius forti succumbere laetho Quam sacra barbaricae spurcanda relinquere genti. 100 Nauticus interea expansis exercitus alis Fluctiuagas refugi sulcando fluminis undas, o funesta dies ! ipsis allabitur oris. Vt primum armati funduntur nauibus hostes, Obsidione premunt huius sublimia circum Moenia coenobii, glomeratis ordine turmis : Primoque aggressu, tentant per tela, per ignes, Pandere ui portas, scalisque ascendere muros. Custodes contra rigidis extrudere contis, Crebraque per patulas tela intorquere fenestras. 110 Ast ubi suffodiunt acta testudine Daci, Tortaque pulsat onus ualidis balista lacertis; Jam uallum, nec terra tumens, nes claustra, nec ipsi Custodes sufficere ualent, nil moenia prosunt : Dant ibi saxa locum, portisque ruentibus, imas Inuadunt arces: pars obuia corpora quaeque Infausta sternunt, nullo discrimine, caede : 
Semirutis alii miscent incendia tectis.

Inclyta Christiparae camerato culmine ad auras

Aetherias surgens exstabat Virginis aedes,

120 Quam ferro accisam quatiunt, et uerbere crebro

Fundamenta cauant: ast uulnere uicta supremo

Ingemuit, traxitque iugo conuulsa ruinam.

Tollitur in coelum densato puluere nubes

Et concussa tremit tam uasto pondere tellus.

Tunc adytum subeunt perfusi membra cruore,

Praedaeque accincti sacrorum fercula, gazas,

Quotquot erant, adimunt; nec ab ulla clade quiescunt, Donec claustra, domos sacrasque a sedibus aedes

Euersas uideant medios considere in ignes.

\section{Eiusdem monasterii restauratio}

130 Interea centum uicibus sol contigit orbem Lanigeri et Librae : tandem cedentibus armis, Pacis alumna Ceres, Martis compescuit iras. Protinus e refugis duo Patres claustra relinquunt Haspreadum repetuntque suum pietatis asylum! Qui cum spectassent, horrendis obsita dumis, Inque uepres redigi, latebrosaque lustra ferarum, Quaeque panomphaeo fuerant sacrata Tonanti, In gemitus toti et lacrymas soluuntur amaras, Singultuque graui uertunt ad sidera uultus. 140 «O Pater, o Bonitas, aiunt, spes unica rerum, Qui tibi confisos nusquam dimittis inanes, Cerne quot excidia et clades, quot funera, casus, Quot mala perpetimur, nostrisque medere ruinis: Jam memores proauis quod nostris foedus inisti, $V t$ sicut Solymum quondam uenerabile templum, Sic ea sacrilega restaures diruta gente, Quae tibi tam celebri sunt structa palatia cultu, $V t$ tibi uota sacro monachorum turba perenni, Munia laudis agat solemnibus aucta triomphis, 150 Priscaque iam uigeat pietas, reuerentia cultus ». Talia perstabant fundentes uota precesque, Thetios uberibus, soloque legumine freti. Nec mora, mittit opem summo qui spectat Olympo Guillelmumque ducem, Rollonis sanguine cretum, Coelitus huc afflat uenando flectere cursum. Qui monachos nactus scitari coepit ab illis, "Quis tam decrepitos istis uos appulit oris? Quaenam religio, aut uestri fiducia uoti? Quae structurae inerant tam uastis conditae eremis, 160 Vnde patent priscae uestigia tanta ruinae?» Cumque rei seriem, a primis annalibus orsi, Ordine narrassent, ex more cibaria praestant. Porrigit alteruter panem, sitientibus offert Alter aquam : siquidem ducebant talibus aeuum. Dux uero aduersus tam uilia fercula, cedit Protinus hisce locis totus profusus in iras. Qui uix ingressus densae penetralia syluae Numinis impulsu Stygium nanciscitur aprum. Qui rapido cursu, rauco bacchatus hiatu, 
170 Vulneris intrepidus transit uenabula, casses, Semianimemque ducem culmis prostrauit acutis. At memor offensae mox uiribus ipse resumptis, Hos repetit Patres, sceleris pro labe pianda: Sumpturusque cibum, quem spreuerat, accubat iisdem Coenobii hujus pollicitus sarcire ruinas.

Tunc fieri delubra iubet cellasque domosque ;

Multaque restituit, priscis subuersa ruinis,

Praedia, diuitias quo possent quaestibus absque, Quique monoptolemi seclusam ducere uitam. 180 Protinus artifices sponsa mercede labori Structuras renouare parant arctando minori Schemate, limitibus domuumque locique tenore Archetypum : tandem fabrefacti encoenia templi, Quae olim Christiparae, rursus sacrantur eidem, 185 Quae gregis huius erit perpes custosque salusque.

B : BnF, coll. diverses, Baluze n ${ }^{\circ}$ 58, fol. 261-266

C : BnF, fr. 4899, fol. 174-176 v.

v. 10 unde monoptolemi... acti : unde monoptolemi quondam bis mille ducenti B \| v. 68 seu : ceu AC || v. 74 fluxu : cursu A || v. 90 gradiui : cradiui AC || v. 118 alii : aliis B || v. 137 sacrata : sacro $C \| v$. 138 gemitus : gemitu $C$

\section{Traduction}

\section{Argument}

Qui que tu sois, voyageur prévoyant, arrête ta marche résolue et regarde ce qui est représenté sur ces merveilleux tableaux. Voici que s'offre à ta vue, comme apparaît d'ordinaire à la surface de l'eau ou sur le miroir parfaitement poli la silhouette de celui qui regarde, les origines illustres de cette lumineuse « gemme » : les anciens ont, en effet, affirmé que Jumièges/Gemmeticus

31 Tu y apprendras quels rois et quels ducs ont concédé tant de biens, tant de terres et de richesses, tant de domaines et de redevances, qui ont permis à neuf cents solitaires, mus par la divine volonté, de pouvoir en cet endroit mener une vie plus étroitement recluse. Quels désastres ont provoqués les escadrons des Lélèges et les bataillons des Daces, et quels hommes enfin ont totalement restauré ces lieux tombés en ruines depuis longtemps, je vais le raconter en rassemblant les faits dans un court poème.

\section{Première fondation du monastère}

Phébus avait déroulé six siècles et huit lustres depuis que la Vierge avait enfanté, quand Philibert, un saint homme, né en Bourgogne celtique d'un illustre lignage, parvint en ce pays et réunit pour vivre dans cette solitude un groupe d'hommes aussi nombreux que celui de Jacob dans le pays de Goshen.

Cette région avait beau être hérissée de forêts envahies par les ronces, cernée de tous côtés de marécages qui l'inondaient, privée d'habitants et de paysans, inconnue de Cérès et du charmant Bacchus, aucun pays cependant ne se montra plus fertile dans le monde entier, après que l'illustre père, grâce au don du roi Dagobert, eut établi les premiers 
fondements de cette sainte église: l'antique aspect fut bientôt, en effet, totalement transformé.

Là où se cachaient les grottes des dieux aux pieds de chèvres, repaires des bêtes solitaires, s'élèvent aujourd'hui des églises étincelantes d'or, réservées à de saints hommes, à l'exemple des temples de l'Olympe étoilé. Nuit et jour désormais des cohortes de moines, tels les chœurs angéliques, répandent leurs prières, font retentir des chants illustres en l'honneur des saints et des chants sacrés en l'honneur du Dieu suprême, le Tonnant, et s'acquittent de leur devoir de louange, précisément là où les bêtes monstrueuses remplissaient en vain les airs de leurs cris horribles.

qui auparavant, funeste et couverte de buissons épineux, nourrissait des êtres chétifs avec les ressources naturelles de la forêt, devint fertile et commença à produire des fruits aussi suaves que l'ambroisie. L'if malodorant, les tilleuls flexibles, l'aconit et la ciguë disparaissent devant les vergers d'Alcinous; la baie noire pousse en grappes et les raisins pourpres grossissent dans les vignes qui deviennent lourdes, tandis que le champ régénéré produit de douces moissons. Là où poussaient roseaux et joncs marécageux prospèrent maintenant des prairies parées de diverses couleurs ; à la place des marécages couverts de roseaux s'étendent désormais de gras pâturages et d'énormes poissons, poussés par les courants, s'échappent de la mer, chaque fois que la fille de Latone, la Titanienne, ramène à l'horizon d'un côté et de l'autre ses chars vermeils. Et le fleuve impétueux reflue à marée montante de sorte que à travers champs et prés verdoyants coulent des rivières qui arrosent la terre et la fertilisent de leurs éléments, à la manière du Nil aux sept branches qui irrigue l'Egypte.

Voilà ce qu'a offert le Créateur de la nature, lui qui fixe un terme au monde, qui maintient la terre ferme sur les immenses océans, qui impose à l'avance aux très grands fleuves leurs limites, pour éviter que Cybèle ne soit recouverte par les ondes gonflées de Thétis. Par ses pluies il apporte l'eau aux montagnes et aux sables stériles, pour que la terre, mère de toutes choses, puisse offrir des dons capables d'assurer l'existence de tous les êtres qui réclament de la nourriture : bêtes qui errent à travers la campagne en friche, oiseaux qui fendent l'air limpide de leurs ailes sifflantes, monstres marins aux formes diverses qui nagent dans l'eau. Tout ce qui vit de l'air éthéré en le respirant, Il vient à son aide et offre des aliments adaptés aux besoins de chacun.

Enfin Celui qui autrefois fit couler l'eau des rochers arides et qui fit tomber du haut du ciel viande et manne, à la manière des averses qui s'abattent du ciel nuageux, pour nourrir, au grand étonnement des cieux, le peuple hébreu qui errait à travers les terres désertiques et les campagnes sauvages, fit de même aux habitants de Jumièges par ses dons généreux de sorte que, malgré les lois de la nature, cette solitude qui avait été autrefois si rude devienne une terre très fertile, entourée par les circonvolutions de la Seine, tout comme l'Ourse est cernée par la queue du serpent sinueux.

\section{Destruction du monastère}

Les astres avaient parcouru dans leur révolution huit siècles et autant de lustres après la naissance du Christ, quand le barbare Hasting, venu des rivages de Dacie, tel un lion avide de nourriture quittant sa tanière, parvint sur le littoral des Belges, au terme d'une guerre des Gètes contre les Francs et soumit les villes des environs et un très grand nombre de places fortes, là où la France porte ses regards vers le nord glacé. Hélas! Malheur! Il 
détruisit les églises où l'on célébrait le Dieu Tonnant qui siège sur un trône élevé, renversant tout par une guerre implacable.

l'estuaire de la Seine : la nouvelle, annonciatrice de si grands désastres, vole rapidement à travers les cités frappées de stupeur. De tous côtés la Neustrie est accablée par des deuils douloureux. De tous côtés les combats la terrassent, les ennemis l'assaillent, l'horreur des armes fond sur elle sous les étendards étincelants du Belliqueux. Les moines s'enfuient au loin : les initiés aux saints mystères gagnent les solitudes retirées d'Haspres, où cloître et demeures monastiques relevaient de notre autorité, pour ne pas être contraints à rompre les vœux sacrés ou de rendre l'âme en abandonnant leurs corps brisés par les supplices cruels à la mort infernale du Styx. Certains cependant résistent en usant de la force : ils garnissent ces lieux de guerriers pour pouvoir repousser les menaces des ennemis. Ils préfèrent succomber à une mort courageuse que d'abandonner les objets sacrés à un peuple barbare qui va les profaner. reflue, ô jour sinistre !, et parvient à ces rives. Dès que les ennemis en armes jaillissent des bateaux, ils encerclent les murs du monastère et les attaquent, après avoir disposé les bataillons en bon ordre: dès le premier choc, ils tentent d'ouvrir les portes de force en lançant des projectiles enflammés et d'escalader les murailles avec des échelles. Les gardes s'efforcent de les repousser avec des perches rigides et de lancer de nombreux traits par l'ouverture des fenêtres.

Mais lorsque les Daces entreprennent de creuser des galeries sous les murs en formant la tortue et que la baliste bandée se met à lancer ses charges par la puissance de ses bras, ni le retranchement, ni la levée de terre, ni l'enceinte, ni les gardes eux-mêmes ne peuvent suffire et les murailles elles-mêmes ne sont d'aucune utilité. Alors les pierres offrent le passage et les ennemis après avoir arraché les portes attaquent la base des points fortifiés. Les uns abattent tous ceux qu'ils rencontrent en causant un affreux massacre sans faire de différence, les autres mettent le feu aux demeures à demi écroulées.

é église de la Vierge mère du Christ dont le faîtage élégant se dressait vers les hauteurs éthérées se tenait encore debout. Ils l'ébranlent après l'avoir attaquée avec le fer et, à coups répétés, ils sapent ses fondations. Vaincue par une ultime blessure, elle gémit et s'écroule, ébranlée jusqu'au sommet. Un épais nuage de poussière s'élève jusqu'au ciel tandis que la terre tremble, percutée par l'énorme masse. Ils pénètrent alors dans l'entrée, les membres couverts de sang, et ils s'empressent de faire du butin en emmenant tous les objets sacrés et tous les trésors qui s'y trouvaient. Ils ne cessent pas leur massacre avant de voir cloitre, demeures et églises saintes entièrement abattus et devenus la proie des flammes.

\section{La restauration du monastère}

Entre temps à cent reprises le soleil atteignit le cercle des constellations du Bélier et de la Balance : avec l'arrêt des violences armées Cérès, la fille de la Paix, contint enfin les colères de Mars. Aussitôt deux des pères qui s'étaient réfugiés à Haspres quittent le monastère et regagnent l'asile de leur vie religieuse. Quand ils eurent découvert que les lieux consacrés au Dieu Tonnant que l'on invoque partout étaient couverts de buissons affreux et redevenus un terrain de broussailles épineuses, où les bêtes sauvages avaient élu domicile, ils se répandirent en gémissements en versant des larmes amères et

Tabularia, Sources en ligne 
tournèrent leur visage vers le ciel dans un lourd sanglot : « $O$ Père de bonté, dirent-ils, unique espoir du monde, Toi qui ne renvoies jamais en vain ceux qui ont mis leur confiance en Toi, vois quelles destructions, quels désastres, quels dommages, quels malheurs et quelles souffrances nous subissons et porte remède à notre anéantissement. Nous nous souvenons aujourd'hui de l'alliance que tu as conclue avec nos anciens, afin que tu restaures, comme tu as restauré le temple vénérable de Salomon, les églises qui ont été détruites par un peuple sacrilège alors qu'elles ont été édifiées en ton honneur pour qu'une foule nombreuses vienne y célébrer ton culte, afin que la foule des moines consacrés à Toi remplisse sans cesse leur devoir de louange par des cérémonies régulières et que l'antique piété refleurisse ainsi que le respect de ton culte. » Tels étaient les vœux et les prières qu'ils présentaient en demeurant en ces lieux et en ne disposant que des secours de Thétis et de légumes.

Sans retard Celui qui du sommet de l'olympe observe le monde envoie son aide. Il inspire au duc Guillaume, issu du sang de Rollon, de dévier durant sa partie de chasse sa course vers cet endroit. Rencontrant les moines il leur demande : "Qui vous a fait aborder sur ces rives, vous qui êtes si vieux? Quelle est votre foi et à quel vœu êtes-vous fidèles? Quelles sont ces constructions qui ont été édifiées dans des solitudes aussi sauvages? Quelle est l'origine de ces vestiges imposants qui témoignent d'une antique destruction?»

Après avoir raconté en détail leur histoire, à partir des premières annales, ils présentent de la nourriture selon la coutume: l'un tend un pain, l'autre offre de l'eau aux hôtes assoiffés, puisque c'est de cela qu'ils vivaient. Mais le duc devant des aliments si ordinaires quitte aussitôt les lieux, en proie à une grande colère. A peine s'était-il enfoncé au cœur de la forêt profonde qu'il se trouve, à l'instigation de la puissance divine, face au sanglier du Styx. L'animal en furie avec des grognements rauques traverse d'une course impétueuse pieux et filets, insensible aux blessures, et, de son groin pointu, il renversa le duc à demi-mort. Celui-ci se souvenant alors de l'offense qu'il avait faite, revient, dès qu'il eut recouvré des forces, vers les pères pour effacer la tache de sa faute: disposé à prendre la nourriture qu'il avait méprisée, il s'installe auprès d'eux et promet de relever le monastère de ses ruines. Il donne l'ordre que soient édifiés églises, cellules et bâtiments. Il restitue les nombreux domaines mis à mal par les antiques destructions et des richesses pour que les solitaires, sans avoir besoin d'en rechercher, puissent mener leur vie à l'écart du monde.

Aussitôt les artisans se préparent à restaurer les édifices, selon le salaire promis pour le travail, en réduisant le projet originel par un plan, par des limites et par une disposition des bâtiments et des lieux plus restreints. Quand ils eurent construit avec art l'église, ils la consacrent de nouveau à la Mère du Christ, à qui elle avait été autrefois dédicacée et qui sera à jamais la gardienne et le salut de ce troupeau. 


\section{NOTES}

1. Le Carmen de fundatione, ruina et restauratione inclyti monasterii Gemmeticensis a été publié dans l'ouvrage d'Arthur DU MONSTIER, Neutria pia seu de omnibus et singulis abbatibus totius Normanniae, Rouen, Berthelin, 1663, en trois parties: 1) argumentum et Prima fundatio dicti monasterii (vers 1-75) : p. 263-264; 2) Destructio eiusdem monasterii (vers 76-129): p. 295-296; 3) De eiusdem restauratione (vers 130-185) : p. 305-306. Jacques-Paul MIGNE a réédité la totalité de ce Carmen dans sa Patrologiae latinae cursus completus, t. 138, Paris, Garnier, 1880, col. 393-398, sous le titre Carmen de monasterio Gemmeticensi, sans aucun changement si ce n'est d'ordre graphique.

2. NORTIER, Michel, « Les sources de l'histoire de Jumièges à la Bibliothèque nationale », Jumièges. Congrès scientifique du XIII centenaire, Rouen, Lecerf, 1955, t. 1, p. 379-395.

3. Ibid.

4. DESHAYES, Charles-Antoine, La Terre gémétique ou notice sur les communes de Jumièges, de Mesnil et d'Yainville, Rouen, P. PÉRIAUX, 1821, p. 53-54.

5. DESHAYES, Charles-Antoine, Histoire de l'abbaye royale de Jumièges, Rouen, Baudry, 1829, p. $192-193$.

6. LANFRY, Georges, "Les bâtiments monastiques de Jumièges: le cloître ", Jumièges. Congrès scientifique du XIII ${ }^{\mathrm{e}}$ centenaire, Rouen, éd. LECERF, t. 2, 1955, p. 499-504.

7. The Gesta Normannorum Ducum of William of Jumièges, Orderic Vitalis and Robert of Torigni, éd. Elisabeth M. C. vAN HOUTS, Oxford, Oxford Clarendon Press, t. 1, 1992, p. XXIX-XXX.

8. Dudon de Saint-Quentin, De moribus et actis primorum Normanniae ducum, éd. Jules LAIR, Caen, Le Blanc-Hardel, 1865 (Mémoires de la Société des Antiquaires de Normandie, t. 23), p. 115-301.

9. ovide, Fastes, 4, 536 ; APOLlinAIRE, Sidoine, Carmina, 9, 210 ; RUFIn, Gregorii orationes, 1, 49, 2.

10. FORTUnAT, Venance, Vita sanctae Radegundis, 21, 51 ; DIACRE, Paul, Vita Gregorii Magni, 2.

11. MIGNE, Jacques-Paul, Patrologiae latinae cursus completus, 153, Paris, éd. MIGNE, 1860, col. 578.

12. OVIDE, Métamorphoses, 11, 198.

13. Cité par Jacqueline DE BOURGOING, Le calendrier maître du temps?, Paris, éd. Gallimard, 2000, p. 133.

14. De l'histoire à la légende: la broderie du Précieux-Sang, Catalogue des Musées municipaux de Fécamp, Fécamp, 2001, p. 44-45.

15. GUILLAUME DE JUMIÈGES, Gesta Normannorum Ducum, I, 5.

16. Ibid.

\section{RÉSUMÉS}

Le Carmen de fundatione, ruina et restauratione inclyti monasterii Gemmeticensis est un poème de 185 vers que présentent trois manuscrits: deux du XVII siècle (Paris, BnF, ms lat. 12778, p. 153-155 et Paris, BnF, ms Baluze n ${ }^{\circ} 58$, p. 174-176 v) et un du début du XVIII ${ }^{\mathrm{e}}$ siècle (Paris, BnF, ms fr. 4899, p. 261-266). 
Ce poème en hexamètres dactyliques évoque la fondation de l'abbaye de Jumièges par l'abbé Philibert, sa destruction par les Vikings et sa restauration par le duc Guillaume Longue Épée vers 940 .

D'après le manuscrit du XVIII siècle, ce poème se trouvait gravé sur des plaques de cuivre fixées à l'entrée du cloître. Comme celui-ci avait été reconstruit vers 1530 par l'abbé François de Fontenay et décoré de fresques illustrant les grandes dates du monastère, il est vraisemblable que ce poème fut composé pour la circonstance afin de faciliter la compréhension des scènes peintes sur les murs du cloître. Traditionnellement daté du $\mathrm{X}^{\mathrm{e}}$, voire du début du $\mathrm{XI}^{\mathrm{e}}$ siècle, ce carmen semble plutôt être un exercice littéraire du milieu du XVI ${ }^{\mathrm{e}}$ siècle comme le montrent certains indices linguistiques

The Carmen de fundatione, ruina et restauratione inclyti monasterii Gemmeticensis is a poem of 185 verses which has survived in three manuscripts: two from the XVIIth century (Paris, BnF, ms lat. 12778, p. 153-155 and Paris, BnF, ms Baluze $\mathrm{n}^{\circ}$ 58, p. 174-176 v) and one from the beginning of the XVIIIth century (Paris, BnF, ms fr. 4899, p. 261-266).

The poem, in dactylic hexameters, describes the foundation of the abbey of Jumièges by Abbot Philibert, its destruction by the vikings, and its restoration by Duke William Longsword in 940 .

According to the XVIIIth-century manuscript the poem had been engraved on copper plates at the cloister's entry. As the cloister had been rebuilt c. 1530 by Abbot Francois de Fontenay and decorated with frescoes illustrating the main occasions in the monastery's history, it is likely that the poem was written at that time in order to faciliate the interpretation of the painings on the cloister walls. Rather than it being a poem of the Xth or beginning of the XIth century, as it has been traditionally dated, it is more likely to be a literary exercise from the middle of the XVIth century as is shown by certain linguistic indications.

\section{INDEX}

Mots-clés : monachisme, Jumièges, poème latin, fondation, restauration, siècle XVIe siècle

Keywords : monasticism, Jumièges, latin poem, foundation, restoration, century 16th century

\section{AUTEUR}

\section{PIERRE BOUET}

Université de Caen Basse-Normandie 